\title{
Linear Free-Enthalpy Relation for Radical Yleld In Fluorescence Quenching by Electron
}

\section{Transfer}

\author{
P. Iwa, U. E. Stelner, E. Vogelmann, and H. E. A. Kramer*
}

Insthut für Physikallsche Chemle der Universität Stuttgart, D-7000 Stuttgart 80, Federal Republic of Germany

The rate constant $\left(k_{\mathrm{q}}\right)$ and net electron-transfer yield $\left(\Phi_{\mathrm{r}}\right)$ in the quenching of an excited oxonine singlet by 24 aromatic amines and methoxy compounds were determined in methanol from stationary fluorescence measurements and flash spectroscopy. The systems investigated correspond to a variation of $\Delta G_{\mathrm{et}}{ }^{\circ}$, the standard free reaction enthalpy of the electron transfer from the quencher to excited singlet oxonine, between -0.12 and $-1.56 \mathrm{eV}$. Whereas the $k_{\mathrm{q}}$ values observed are all close to the diffusion controlled limit, $\Phi_{\mathrm{r}}$ increases when $\Delta G_{\mathrm{et}}{ }^{\circ}$ becomes more negative, whereby the following linear correlation is observed: $\ln \Phi_{\mathrm{r}}=-5.7-\left(3.2 \mathrm{eV}^{-1}\right) \Delta G_{\mathrm{et}}^{\circ}$. The implications of this relationship on the mechanism of free-radical formation and electronic deactivation are discussed. Mechanisms where either a solvent-shared radical pair or a relaxed exciplex are assumed as exclusive intermediates of the quenching reaction are shown to be incompatible with the experimental result. It is suggested that in the quenching reaction radical pairs and exciplexes are formed via a nonrelaxed charge-transfer state, the branching ratio between radical pair and exciplex formation depending on $\Delta G_{\mathrm{et}}{ }^{\circ}$.

\section{Introduction}

Electron-transfer reactions with electronically excited states are of considerable recent interest, especially with respect to their potential application in solar-energy storage. ${ }^{1}$ Studies on fluorescence quenching have greatly

(1) N. N. Lichtin in "Solar Power and Fuels", J. R. Bolton, Ed., Academic Press, New York, 1977, p 119. contributed to the understanding of such reactions. ${ }^{2}$ Whereas in unpolar solvents electron donor-acceptor interaction usually leads to the formation of fluorescing excited charge-transfer complexes (exciplexes), the formation of free-radical ions can be detected in polar sol-

(2) See, for example "Modern Fluorescence Spectroscopy", Vols. 1 and 2, E. L. Wehry, Ed., Plenum Press, New York, 1976. 
Scheme I

$$
\begin{aligned}
& A^{*}+0 \frac{k_{12}}{k_{21}} A^{[1]} A^{*}-D \frac{k_{23}}{k_{32}}{ }^{\prime}\left(A^{*}-D^{*}, 1,\right.
\end{aligned}
$$

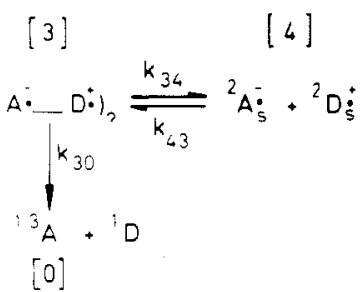

vents. In this case electron-transfer reactions with excited states are characterized by two parameters: the rate constant $k_{\mathrm{q}}$, at which the excited state is quenched, and the net yield $\Phi_{r}$ of electron transfer, which is usually lower than $100 \%$ due to several decay processes competing with the formation of free radicals from the primary electrontransfer product.

As was shown by Rehm and Weller, ${ }^{3}$ the rate constant $k_{\mathrm{q}}$ of excited singlet quenching by an electron-transfer mechanism is determined by the standard free-enthalpy change $\Delta G_{\mathrm{et}}{ }^{\circ}$ of the corresponding electron-transfer reaction. The characteristic Rehm-Weller relation between $\log k_{\mathrm{q}}$ and $\Delta G_{\mathrm{et}}{ }^{\circ}$ has been found to apply also to electron-transfer reactions with excited triplet states of dyes ${ }^{4}$ and transition-metal complexes. ${ }^{5}$ In contrast to $k_{\mathrm{q}}$, for the net yield $\Phi_{\mathrm{r}}$ of electron transfer such a general correlation does not exist.

A general mechanistic framework describing the excited-state quenching and free-radical formation is presented in Scheme I, which is a generalization of the scheme originally used by Rehm and Weller. ${ }^{3}$ In dilute solutions the excited molecule (here acting as an electron acceptor A) and the electron donor-acceptor counterpart (here electron donor $\mathrm{D}$ ) have to undergo diffusive encounters (stage 2) where at some encounter distance, symbolized by "-" electron transfer occurs to give the primary electrontransfer product $\left({ }^{1}\left(\mathrm{~A}^{-}--\mathrm{D} \cdot{ }^{+}\right)_{\text {, }}\right.$, stage 3 where the question mark index denotes that we leave for the moment unspecified whether this species is relaxed with respect to solvation and whether there is intermolecular interaction between $\mathrm{A}^{-}$and $\mathrm{D}^{+}$other than electrostatic Coulomb interaction.

The primary electron-transfer intermediate [3] will dissociate at some stage of solvent relaxation into the free solvated ion radicals [4], a process that has to compete with deactivation processes involving electron retransfer from the acceptor to the donor to yield electronically lower states (singlet ground state of D and A or locally excited triplet state of either A or D), which in effect represent free-energy-loss channels and may be either undesired, e.g., in solar-energy storage applications, or welcome, e.g., in light stabilization applications.

(3) D. Rehm and A. Weller, Ber, Bunsenges. Phys. Chem., 73, 834 (1969).

(4) (a) E. Vogelmann, S. Schreiner, W. Rauscher, and H. E. A. Kramer, Z. Phys. Chem. (Wiesbaden), 101, 321 (1976). (b) L. W. Romaschov, Yu. I. Kirjuchin, and Ch. S. Bagdasajan, Dokl. Akad. Nauk SSSR, 230, 1145 (1976). (c) S. Tamura, K. Kikuchi, and H. Kokubun, Z. Phys. Chem (Wiesbaden), 111, 7 (1978). (d) E. Vogelmann, W. Rauscher, and H. E. A. Kramer, Photochem. Photobiol. 29, 771 (1979). (e) E. Vogelmann, W. Rauscher, R. Traber, and H. E. A. Kramer, Z. Phys. Chem. (Wiesbaden) 124, 13 (1981)

(5) (a) R. Ballardini, G. Varani, M. T. Indelli, F. Scandola, and V. Balzani, J. Am. Chem. Soc., 100, 7219 (1978). (b) V. Balzani, F. Bollette F. Scandola, and R. Ballardini, Pure Appl. Chem., 51, 299 (1979). (c) D. G. Whitten, Acc. Chem. Res., 13, 83 (1980).
According to Scheme I the free-radical yield per excited molecule quenched can be expressed as

$$
\Phi_{\mathrm{r}}=k_{34} /\left(k_{30}+k_{34}\right)
$$

and it is clear that $\Phi_{\mathrm{r}}$ is influenced by as many factors as the rate constants $k_{30}$ and $k_{34}$. For instance it has been shown that $k_{34}$ depends critically on the solvent viscosity, ${ }^{6}$ and on the solvent polarity if [3] is a radical ion pair with Coulombic interaction. ${ }^{7}$ On the other hand the rate constant $k_{30}$ depends on the energy gap between species [3] and [0] (energy gap law of radiationless transitions ${ }^{8}$ ) and on spin-orbit coupling ${ }^{9}$ or hyperfine coupling properties $^{10}$ if [3] $\rightarrow[0]$ is multiplicity forbidden.

It is clear that whenever a simple relation between one single physical parameter of the donor-acceptor-solvent system and the free radical yield $\Phi_{\mathrm{r}}$ should be observable, the parameter in question should interfere as little as possible with the other parameters which may influence $k_{30}$ or $k_{34}$. Therefore such a relation cannot be expected to hold in general. Thus Hino et al. ${ }^{11}$ when trying to correlate the free-energy change of the electron-transfer step between pyrene in its excited singlet state and various quenchers (donors and acceptors) with the radical yield, did not observe a systematic correlation. On the other hand results of the same group on the ionic dissociation yield, when exciting 1,2,4,5-tetracyanobenzene and pyromellitic dianhydride ground-state electron donor-acceptor complexs into their charge-transfer band, indicate a general tendency of the radical yield to increase with the energy of the excited charge-transfer state, ${ }^{12}$ which was thought to reflect the energy gap law effect on $k_{30}$.

In this paper we want to report results on the radical yield in the quenching of oxonine fluorescence by a series of electron donors in methanol where a distinct correlation between $\Phi_{\mathrm{r}}$ and $\Delta G_{\mathrm{et}}{ }^{\circ}$ is observed. These results allow interesting conclusions to be drawn concerning the behavior of the primary electron-transfer product [3] as a function of $\Delta G_{\mathrm{et}}{ }^{\circ}$

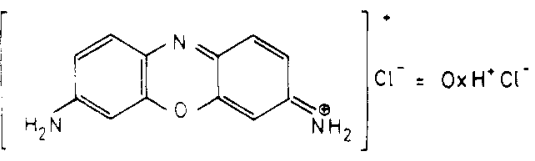

The cationic dye oxonine (formula $\mathrm{OxH}^{+}$) is a heteroanalogue of thionine that has been intensively studied in electron-transfer reactions involving the excited triplet state; cf. ref 4a. Due to the decrease of spin-orbit coupling when substituting sulfur in thionine by oxygen, ${ }^{13}$ oxonine has a very low quantum yield of spontaneous triplet formation $\left(<10^{-2}\right), 14$ making it ideally suited for studies of excited singlet state reactions with this class of dyes. ${ }^{15}$

(6) G. Winter and U. Steiner, Ber. Bunsenges. Phys. Chem., 84, 1203 $(1980)$

(7) T. Hino, H. Masuhara, and N. Mataga, Bull. Chem. Soc. Jpn. 49, 394 (1976)

(8) K. F. Freed, Top. Appl. Phys., 15, 23 (1976).

(9) E. Vogelmann and H. E. A. Kramer, Photochem. Photobiol., 24, 595 (1976)

(10) (a) K. Schulten, H. Staerk, A. Weller, H.-J. Werner, and B. Nickel, Z. Phys. Chem. (Wiesbaden), 101, 371 (1976). (b) R. Haberkorn and M. E. Beyerle, Z. Naturforsch. A, 31a, 499 (1976). (c) M. E. Beyerle, R. Haberkorn, W. Bube, E. Steffens, H. Schröder, H. J. Neusser, E. W. Schlag, and H. Seidlitz, Chem. Phys., 17, 139 (1976)

(11) T. Hino, H. Akazawa, H. Masuhara, and N. Mataga, J. Phys. Chem., 80, 33 (1976)

(12) J. Hinatu, F. Yoshida, H. Masuhara, and N. Mataga, Chem. Phys. Lett., 59, 80 (1978).

(13) E. Vogelmann, H. Schmidt, U. Steiner, and H. E. A. Kramer, Z. Phys. Chem. (Wiesbaden), 101, 321 (1976)

(14) R. Föll, Universität Stuttgart, to be published. 
Since $\mathrm{OxH} \mathrm{H}^{+}$is discharged in an electron-transfer reaction, where it acts as an electron acceptor, there will be no Coulomb attraction in the radical pair produced. Therefore $k_{34}$ should be fairly high so that sizable and accurately measurable quantum yields of free-radical formation are to be expected for a large range of $k_{30}$ values.

For a monopositive acceptor ion and a neutral electron donor, the standard free enthalpy $\Delta G_{\text {et }}{ }^{\circ}$ of electron transfer is given by ${ }^{3}$

$$
\Delta G_{\mathrm{et}}{ }^{0}=\mathcal{F} E\left(\mathrm{D} / \mathrm{D}^{+}\right)-\mathcal{F} E\left(\mathrm{~A} \cdot / \mathrm{A}^{+}\right)-\Delta E_{00}\left({ }^{1 *} \mathrm{~A}^{+}\right)
$$

where $\mathcal{F}$ is Faraday's constant, $E\left(\mathrm{D} / \mathrm{D}^{+}\right)$and $E\left(\mathrm{~A} \cdot / \mathrm{A}^{+}\right)$ denote the redox potentials of donor and acceptor, respectively, and $\Delta E_{00}\left({ }^{1 *} \mathrm{~A}^{+}\right)$is the purely electronic excitation energy of the first excited singlet state of the acceptor. Since the acceptor is not changed in the present experiments, variation of $\Delta G_{\mathrm{et}}{ }^{\circ}$ is exclusively due to $E$ $\left(\mathrm{D} / \mathrm{D} \cdot{ }^{+}\right)$.

Considering the relatively large discrepancies between polarographic oxidation potentials determined by different authors, we thought it important to apply a large set of donors that have been polarographically investigated in the same laboratory under the same experimental conditions, so that, at least on a relative scale, the potentials obtained should be reliable. The donors chosen were aromatic amines and methoxy compounds, the oxidation potentials of which have been determined by Zweig et al. ${ }^{16}$

\section{Experimental Section}

Apparatus. The conventional flash apparatus was the same as described by Kramer and Maute. ${ }^{17}$ The halfduration of the exciting flash was about $5 \mu \mathrm{s}$, and the cuvette was surrounded by a Kodak Wratten gelatin filter No. 8, which absorbs flash light of wavelength shorter than $400 \mathrm{~nm}$, so that the dye was excited only in its first absorption band and an excitation of the quenching compounds was also excluded.

Oxygen was removed by bubbling nitrogen $\left(\mathrm{O}_{2}\right.$ content lower than $6 \mathrm{ppm}$ ) through the methanolic solution for 20 min. Fluorescence and absorption measurements were carried out on a Fluorispec SF1 and a Zeiss DMR 10 spectrophotometer, respectively.

Chemicals. Methanol (Merck p.a.) was used as solvent for all measurements. Oxonine was prepared by Vogelmann according to a procedure described in ref 18 . The commercially available electron donors $N, N, N^{\prime}, N^{\prime}$-tetramethyl-p-phenylenediamine (TMPDA, EGA), $N, N, N^{\prime}$,$N^{\prime}$-tetramethylbenzidine ( $\left.N, N, N^{\prime}, N^{\prime}-\mathrm{TMB}, \mathrm{EGA}\right), N, N$ diethylaniline (DEA, Fluka), $N, N$-dimethylaniline (DMA, Fluka), 1,2-dimethoxybenzene (1,2-DMB, Fluka), 1,4-dimethoxybenzene (1,4-DMB, Fluka), 1,2,3-trimethoxybenzene (1,2,3-TMB, Aldrich), 1,2,4-trimethoxybenzene (1,2,4-TMB), 1,3,5-trimethoxybenzene (1,3,5-TMB, Aldrich), 1-methoxynaphthalene (1-MN, EGA), 2-methoxynaphthalene (2-MN, EGA), and 3,3'-bis(dimethoxy)biphenyl (3,3'-BDMBPh, EGA) were purified by distillation, sublimation, or recrystallization and stored under argon.

The tertiary amines 3,4-dimethoxy- $N, N$-dimethylaniline (3,4-DMDMA), 2,4-dimethoxy- $N, N$-dimethylaniline (2,4-

(15) (a) E. Vogelmann and H. E. A. Kramer, Photochem. Photobiol. 23, 383 (1976). (b) R. Bonneau and J. Joussot-Dubien, Z. Phys. Chem. (Wiesbaden), 101, 225 (1976). (c) R. Bonneau, Photochem. Photobiol., 25, 129 (1977).

(16) (a) A. Zweig, W. G. Hodgson, and W. Jura, J. Am. Chem. Soc 86, 4124 (1964). (b) A. Zweig, J. E. Lancaster, M. T. Neglia, and W. Jura ibid., 86, 4130 (1964). (c) A. Zweig, A. Mauser, and B. Roberts, J. Org. Chem., 32, 1322 (1967).

(17) H. E. A. Kramer and A. Maute, Photochem. Photobiol., 15, 7 (1972).

(18) F. Kehrmann and A. Saager, Chem. Ber., 36, 475 (1903).
DMDMA), $p$-methoxy- $N, N$-dimethylaniline (p-MDMA), $m$-methoxy- $N, N$-dimethylaniline ( $m$-MDMA), diphenylmethylamine (DPhMA), and 1,5-bis(dimethylamino)naphthalene (1,5-BDMAN) were prepared according to Zweig et al. ${ }^{16 \mathrm{~b}}$ from the corresponding commercially available primary or secondary amines (all EGA).

Tetramethyl-o-phenylenediamine (TMODA) and tetramethyl- $m$-phenylenediamine (TMMDA) were prepared as described in the literature. ${ }^{19}$ All tertiary amines were purified by sublimation or distillation. In the latter case the purified donors were frozen with liquid nitrogen and then saturated with argon during thawing before removing the material from the distillation apparatus.

The methoxyaromatics 1,4-dimethoxynaphthalene (1,4-DMN), 1,5-dimethoxynaphthalene (1,5-DMN), 1,8dimethoxynaphthalene (1,8-DMN), and 4-methoxybiphenyl (4-MBPh) were prepared by Rauscher ${ }^{20}$ from the corresponding commercially available hydroxy compounds.

\section{Determination of Quenching Parameters}

The processes to be considered in our experiments are summarized in eq $3 a-e$. By absorption of light the oxonine

$$
\begin{aligned}
& \mathrm{OxH}^{+} \stackrel{I_{\text {㭊 }}}{\longrightarrow} 1 * \mathrm{OxH}^{+} \\
& 1 * \mathrm{OxH}^{+} \stackrel{k_{\mathrm{d}}}{\longrightarrow} \mathrm{OxH}^{+} \\
& { }_{1 *} \mathrm{OxH}^{+} \stackrel{k_{\mathrm{f}}}{\longrightarrow} \mathrm{OxH}^{+}+h \nu \\
& 1 * \mathrm{OxH}^{+}+\mathrm{D} \stackrel{k_{x}}{\longrightarrow} \cdot \mathrm{OxH}+\mathrm{D}^{+} \\
& { }^{1} * \mathrm{OxH}^{+}+\mathrm{D} \stackrel{k_{g}}{\longrightarrow} \mathrm{OxH}^{+}+\mathrm{D}
\end{aligned}
$$

ground state $\left(\mathrm{OxH}^{+}\right)$is converted to its lowest excited singlet state $\left({ }^{1 *} \mathrm{OxH}^{+}\right)$, which may undergo radiationless transition to the ground state (eq $3 \mathrm{~b}$ ) or emit a fluorescence photon (eq $3 \mathrm{c}$ ). The rate constants $k_{\mathrm{d}}$ and $k_{\mathrm{f}}$ may be combined to give the effective monomolecular decay constant $k_{0}$ of the excited singlet (eq 4). The interaction of

$$
k_{0}=k_{\mathrm{d}}+k_{\mathrm{f}}
$$

the excited singlet state with the donor may result in formation of free radicals (eq $3 d$ ) or bimolecular deactivation to the ground state (eq $3 \mathrm{e}$ ), whereby the observed quenching rate constant $k_{\mathrm{q}}$ is given by

$$
k_{\mathrm{q}}=k_{\mathrm{r}}+k_{\mathrm{g}}
$$

(a) Determination of Quenching Constants. The quenching constants $k_{\mathrm{q}}$ were determined by measuring the relative fluorescence intensity of oxonine as a function of the donor concentration. So that sufficient fluorescence quenching could be obtained, donor concentrations up to $\approx 0.05 \mathrm{M}$ had to be used. In this range of concentration, however, time-dependent diffusion in the quenching process must not be neglected. Therefore the quenching rate constants $k_{\mathrm{q}}$ had to be evaluated by a modified SternVolmer procedure as described by Weller. ${ }^{21}$ It is based on eq 6, where $I$ and $I_{0}$ are relative fluorescence intensities

$$
I / I_{0}=\exp \left(V_{\mathrm{D}}\left(I / I_{0}\right)^{1 / 2} c\right) /\left(1+k_{\mathrm{q}} \tau_{0} c\right)
$$

with and without quencher, $c$ is the quencher concentration, $\tau_{0}$ is the oxonine singlet lifetime in the absence of a quencher (3.5 $\mathrm{ns}$ in methanol), and $V_{\mathrm{D}}$ is an effective reaction volume, to be determined, according to Weller. ${ }^{21}$ With use of eq 6 the experimentally observed $I / I_{0}$ vs. $c$

(19) S. F. Torf and N. V. Khromov-Borisov, Zh. Obhsch. Khim., 30, $1798(1960)$.

(20) W. Rauscher, Thesis, Universität Stuttgart 1978, Stuttgart

(21) A. Weller, Z. Phys. Chem. (Wiesbaden), 13, 353 (1957). 
curve was fitted in a computer calculation by a suitable choice of $k_{\mathrm{q}}$.

(b) Determination of Radical Yields. From eq 3a-e the radical yield per excited singlet state produced in a flash experiment is given by

$$
\Phi_{\mathrm{r},[\mathrm{D}]}=k_{\mathrm{r}}[\mathrm{D}] /\left(k_{0}+k_{\mathrm{q}}[\mathrm{D}]\right)
$$

where the symbol $\Phi_{r,[D]}$ denotes that this quantity depends on the donor concentration. We are, however, interested in the ratio $k_{\mathrm{r}} / k_{\mathrm{q}}$, which is the limiting value obtained when $\Phi_{r,[D]}$ is extrapolated to infinite donor concentration.

$$
\Phi_{\mathrm{r}}=\lim _{[\mathrm{D}] \rightarrow \infty} \Phi_{\mathrm{r},[\mathrm{D}]}=k_{\mathrm{r}} / k_{\mathrm{q}}
$$

$\Phi_{r,[D]}$ may be directly related to $\Phi_{r}$ by the use of relative fluorescence quantum yields. The fluorescence quantum yield $\Phi_{f, 0}$ in donor-free solutions and $\Phi_{f,[D]}$ in the presence of some donor concentration are given by

$$
\begin{gathered}
\Phi_{\mathrm{f}, 0}=k_{\mathrm{f}} / k_{0} \\
\Phi_{\mathrm{f},[\mathrm{D}]}=k_{\mathrm{f}} /\left(k_{0}+k_{\mathrm{q}}[\mathrm{D}]\right)
\end{gathered}
$$

Combining these two equations yields

$$
\frac{k_{0}+k_{\mathrm{q}}[\mathrm{D}]}{k_{\mathrm{q}}[\mathrm{D}]}=\frac{1}{1-\Phi_{\mathrm{f},[\mathrm{D}]} / \Phi_{\mathrm{f}, 0}}
$$

an expression depending on the relative fluorescence quantum yield $\Phi_{f,[D]} / \Phi_{f, 0}$ only. From eq 11 we can relate $\Phi_{\mathrm{r}}$ and $\Phi_{\mathrm{r},[\mathrm{D}]}$ by the relative fluorescence quantum yield:

$$
\Phi_{r}=\Phi_{r,[D]} \frac{1}{1-\Phi_{f,[D]} / \Phi_{f, 0}}
$$

The actual radical yield $\Phi_{f,[D]}$ at a given donor concentration is defined experimentally by eq 13 , where

$$
\Phi_{\mathrm{r},[\mathrm{D}]}=\frac{[\cdot \mathrm{OxH}]_{0}}{\left[{ }^{1 *} \mathrm{OxH} \mathrm{xH}_{0}\right.}=\frac{E^{\lambda} \cdot \mathrm{OxH}}{\epsilon^{\lambda} \cdot \mathrm{OxH} d\left[{ }^{1 *} \mathrm{OxH}^{+}\right]_{0}}
$$

$\left[{ }^{1 *} \mathrm{OxH}^{+}\right]_{0}$ stands for the hypothetical total concentration of all excited dye molecules produced in the flash experiment and $[\cdot 0 \mathrm{xH}]_{0}$ stands for the total concentration of all semiquinone radicals produced in the same experiment. The latter is obtained from the absorbance $E^{\lambda} .0 \times \mathrm{xH}$ of the semiquinone radical shortly after the photolysis flash when the loss of these radicals is still negligible, since they decay in the millisecond range. With a constant flash intensity in a series of experiments and depletion of only a small fraction of the ground state, the quantity $\left[{ }^{1 *} \mathrm{OxH}\right]_{0}$ is constant and $E^{\lambda}$.0xH provides a relative measure of $\Phi_{r,[D]}$ So that absolute quantum yields could be obtained, $\left[{ }^{1 *} \mathrm{OxH}^{+}\right]_{0}$ was determined, with induced triplet formation by allylthiourea as a quantum standard reaction for which a quantum yield of $0.3 \pm 0.1^{22}$ has been estimated. In typical experiments $\left[{ }^{1 *} \mathrm{OxH}^{+}\right]_{0}$ was about equal to the total dye concentration, meaning that on an average each molecule was excited once. Since the singlet lifetime, however, is very much shorter than the flash duration the momentary bleaching of the dye ground state never exceeded the final radical yield throughout the experiment. The observation of the radical yield was made at $403 \mathrm{~nm}$, where the molar extinction coefficient $\epsilon^{403} \cdot{ }_{0 x H}$ of $\cdot 0 x H$ is $15000 \mathrm{M}^{-1} \mathrm{~cm}^{-1}$ (see below). The optical path length $d$ of the flash cuvette was $10 \mathrm{~cm}$.

\section{Results}

Absorption Spectrum of Oxonine Semiquinone. In order to determine the radical yield in the quenching of

(22) R. E. Föll, Diplomarbeit, Universität Stuttgart, 1976, Stuttgart.

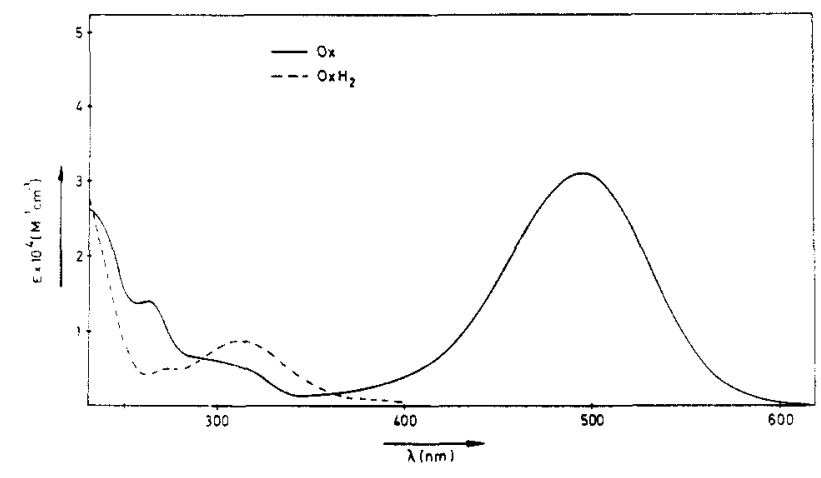

Flgure 1. Spectra of oxonine (-) and leucooxonine (---), both $2 \times$ $10^{-5} \mathrm{M}$ in methanol containing $5 \times 10^{-2} \mathrm{M}$ sodium methylate. The protolytic forms present are $\mathrm{Ox}$ and $\mathrm{OxH}_{2}$, respectively.

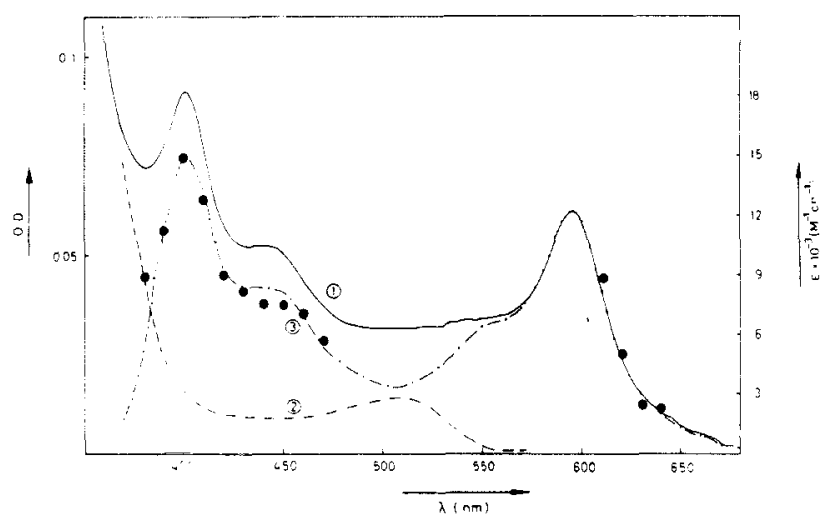

Figure 2. Determination of the absorption spectrum of semioxonine (3) $(\cdot 0 \times \mathrm{H})$. The extinction coefficient is given on the right-hand side of the vertical axis. Spectrum 1 is obtained for a mixture made up from $2.5 \times 10^{-6} \mathrm{M}$ oxonine and $5 \times 10^{-5} \mathrm{M}$ leucooxonine in methanol containing $5 \times 10^{-2} \mathrm{M}$ sodium methylate. Spectrum 2 is obtained for $4.75 \times 10^{-5} \mathrm{M}$ leucooxonine in the same solvent. Spectrum 3 results as the difference of spectra 1 and 2 (()) indicates transient absorption obtained on flashing a solution of $2 \times 10^{-5} \mathrm{M}$ oxonine and $1 \times 10^{-1}$ $M$ DEA in oxygen-free methanol; the latter is normalized to the maximum of semioxonine (.OxH) absorption).

$1 * \mathrm{OxH}^{+}$by some electron donor it was desirable to have detailed information on the absorption spectrum of the radical $.0 x H$. Actually it turned out that in oxygen-free, alkaline solutions a comproportionation equilibrium between deprotonated oxonine $(\mathrm{Ox})$ and leucooxonine $\left(\mathrm{OxH}_{2}\right)$ was established with a semiquinone formation constant $K .0 x H$ large enough to achieve a considerable fraction of conversion into the semiquinone radicals.

$$
\mathrm{Ox}+\mathrm{OxH}_{2} \rightleftarrows 2 . \mathrm{OxH}
$$

A suitable condition for having all the redox forms involved in equilibrium 14 present in the protolytic forms indicated was realized when $5 \times 10^{-2} \mathrm{M}$ sodium methylate solutions were used. Formation of the radical $\cdot \mathrm{OxH}$ in mixtures of oxygen-free solutions of $\mathrm{Ox}$ and $\mathrm{OxH}_{2}$ is indicted by the appearance of two peaks, at 403 and $595 \mathrm{~nm}$, which are neither present in the pure $\mathrm{Ox}$ nor the pure $\mathrm{OxH}_{2}$ spectrum (Figure 1). Analyzing the spectrum of the $\mathrm{Ox} / \mathrm{OxH}_{2}$ mixture as a function of the mixing ratio yields the semiquinone formation equilibrium constant (eq 15) $K_{.0 x \mathrm{H}}$

$$
K_{. \mathrm{OxH}}=\frac{[\cdot \mathrm{OxH}]^{2}}{[\mathrm{Ox}]\left[\mathrm{OxH}_{2}\right]}
$$

to be 7.3. From this value it is obtained that in a mixture made up of $[\mathrm{Ox}]=2.5 \times 10^{-6} \mathrm{M}$ and $\left[\mathrm{OxH}_{2}\right]=5 \times 10^{-5}$ $\mathrm{M}$, conversion to. $\mathrm{OxH}$ is almost quantitative.

Figure 2 shows how with such a solution the spectrum of pure $\cdot \mathrm{OxH}$ is obtained by correcting the spectrum of the 
TABLE I: Quantum Yields $\Phi_{r}$ and Quenching Rate Constants $k_{\mathrm{q}}$ for Fluorescence Quenching of Oxonine by Various Electron Donors in Methanol

\begin{tabular}{|c|c|c|c|c|}
\hline donor ${ }^{a}$ & $\Phi_{Y}^{b}$ & $E\left(\mathrm{D} / \mathrm{D}^{++}\right)^{c}$ & $\begin{array}{c}-\Delta G_{\text {et }}^{\circ} \\
e V^{d}\end{array}$ & $\begin{array}{l}k_{\mathrm{q}}, 10^{9} \\
\mathrm{M}^{-1} \mathrm{~s}^{-1}\end{array}$ \\
\hline 1. TMPDA & 0.45 & 0.16 & 1.56 & 18 \\
\hline 2. 3,4-DMDMA & 0.19 & 0.43 & 1.29 & 16 \\
\hline 3. $N, N, N^{\prime}, N^{\prime}-\mathrm{TMB}$ & 0.18 & 0.43 & 1.29 & 18 \\
\hline 4. 2,4-DMDMA & 0.10 & 0.53 & 1.19 & 13 \\
\hline 5. TMODA & 0.16 & 0.54 & 1.18 & 17 \\
\hline 6. TMMDA & 0.17 & 0.58 & 1.14 & 16 \\
\hline 7. $1,5-\mathrm{BDMAN}$ & 0.15 & 0.58 & 1.14 & 13 \\
\hline 8. $p$-MDMA & 0.13 & 0.59 & 1.13 & 16.5 \\
\hline 9. $m$-MDMA & 0.065 & 0.75 & 0.97 & 17 \\
\hline 10. DEA & 0.072 & 0.76 & 0.96 & 19 \\
\hline 11. DMA & 0.037 & 0.79 & 0.93 & 15.1 \\
\hline 12. DPhMA & 0.058 & 0.84 & 0.88 & 15.5 \\
\hline 13. 1,4-DMN & 0.03 & 1.10 & 0.62 & 10.8 \\
\hline 14. 1,2,4-TMB & 0.013 & 1.12 & 0.60 & 10 \\
\hline 15. 1,8-DMN & 0.019 & 1.17 & 0.55 & 10.8 \\
\hline 16. 1,5-DMN & 0.015 & 1.28 & 0.44 & 9.6 \\
\hline 17. 1,4-DMB & 0.0098 & 1.34 & 0.38 & 9.5 \\
\hline 18. 1-MN & 0.014 & 1.38 & 0.34 & 9 \\
\hline 19. $1,2,3$-TMB & 0.0073 & 1.42 & 0.30 & 7 \\
\hline 20. $1,2-\mathrm{DMB}$ & 0.008 & 1.45 & 0.27 & 8.4 \\
\hline 21. $1,3,5$-TMB & 0.0046 & 1.49 & 0.23 & 7 \\
\hline 22. $2-\mathrm{MN}$ & 0.0046 & 1.52 & 0.20 & 8 \\
\hline 23. 4-MBPh & 0.0046 & 1.53 & 0.19 & 6.6 \\
\hline 24. $3,3^{\prime} \cdot \mathrm{BDMBPh}$ & 0.0058 & 1.60 & 0.12 & 9 \\
\hline
\end{tabular}

${ }^{a}$ See the Experimental Section for an explanation of abbreviations. $b$ Relative accuracy $\pm 5 \%$, absolute accuracy $\pm 30 \%$. c From ref 16 , vs. SCE in acetonitrile. Corrections for a solvent change from acetonitrile to methanol according to Born ${ }^{25}$ are in the order of $0.01 \mathrm{~V}^{20}$ ${ }^{d}$ Calculated from eq 2 with $E_{00}\left({ }^{1}{ }^{*} \mathrm{OxH}^{+}\right)=2.07 \mathrm{eV}^{20}$ and $E\left(\mathrm{~A} \cdot / \mathrm{A}^{+}\right)=-0.35 \mathrm{~V}$ vs. SCE in methanol. ${ }^{22}$

mixture for the absorption of the unreacted leucooxonine $\mathrm{OxH}_{2}$. The absorption coefficients in the maxima of the . OxH spectrum are $\epsilon_{403}=15000$ and $\epsilon_{595}=12500 \mathrm{M}^{-1} \mathrm{~cm}^{-1}$. In pure methanol the dye is present in the form $\mathrm{OxH}^{+}$, which in the excited singlet quenching by electron donors should be transformed to the radical .OxH.

A typical transient spectrum obtained when a methanolic solution of oxonine $\left(\mathrm{OxH}^{+}\right)$is flashed together with an electron donor is shown in Figure 2. The short-wavelength absorption, around $400 \mathrm{~nm}$, is almost identical in shape with that obtained for $.0 \mathrm{xH}$ under stationary conditions, whereas with many of the donors applied, the long-wavelength absorption (obtainable only where it does not overlap too strongly the ground-state absorption of $\mathrm{OxH}^{+}$) is somewhat enhanced with respect to the long wavelength absorption in the stationary $.0 \times H$ spectrum, which may be attributed to an absorption of the donor radical. ${ }^{23,24}$ No changes in the shape of the short-wavelength absorption band were to be observed with the present donors, so it was assumed that the absorption of the corresponding donor radicals is negligible in this part of the spectrum. From the transient absorption produced at $403 \mathrm{~nm}$ and the extinction coefficient of $.0 \times \mathrm{H}$ at this wavelength, the radical yield was determined as described above.

Quenching Rate Constants and Radical Yields. In Table I are listed the data pertinent to the dynamic quenching of singlet excited oxonine $\left({ }^{1 *} \mathrm{OxH}^{+}\right)$by 24 aromatic amines and methoxy compounds. Given are the quenching rate constants $k_{\mathrm{q}}$, the yields of free radicals $\Phi_{\mathrm{r}}$, equivalent to the net yield of electron transfer in the

(23) G. N. Lewis and D. Lipkin, J. Am. Chem. Soc., 64, 2801 (1942); T. Shida, W. H. Hamill, J. Chem. Phys., 44, 2369 (1966).

(24) W. Aichele, Diplomarbeit, Universität Stuttgart, 1975, Stuttgart.

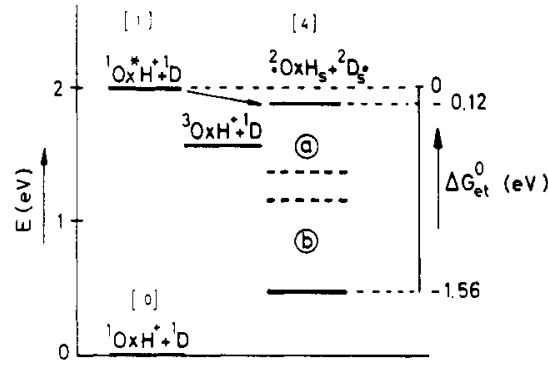

Figure 3. Energy level diagram indicating the relative energles of the exclted oxonine singlet and triplet and the range of energles of the radial pairs produced in the flash experiments with various donors $D$. The square brackets refer to Scheme I; the radical-pair energy region is denoted a for $D=$ aromatic methoxy compound and $b$ for $D=$ aromatic amine.

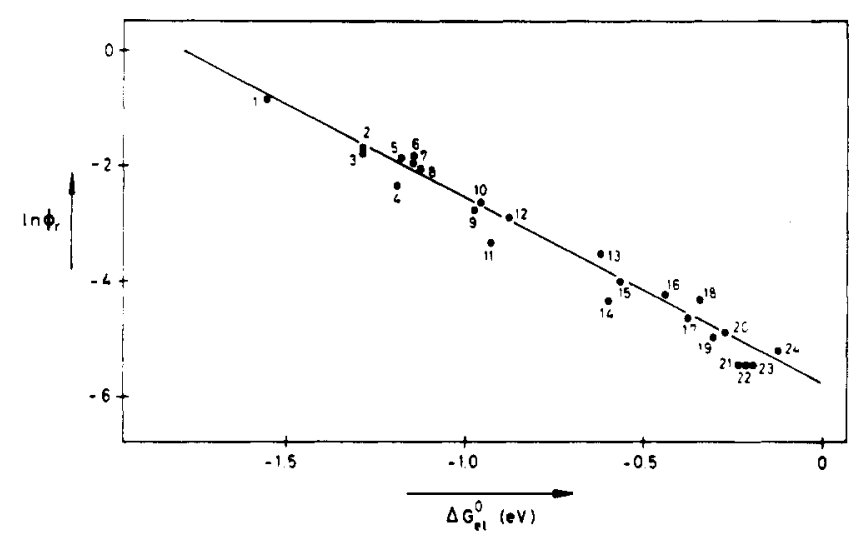

Figure 4. Plot of in $\Phi_{r}$ vs. $\Delta G^{\circ}$. The numbers in the diagram refer to the donors listed in Table I.

quenching reaction, the oxidation potentials $E\left(\mathrm{D} / \mathrm{D}^{+}\right)$of the donors, and the free-enthalpy change $\Delta G_{\mathrm{et}}{ }^{\circ}$ of the electron-transfer reaction with the excited oxonine singlet. An energy-level diagram is shown in Figure 3. The free-enthalpy range investigated extends over $1.44 \mathrm{eV}$ and is completely located in the negative region. Thus it is not surprising that, in agreement with the original findings of Rehm and Weller, all rate constants are practically diffusion controlled. Nevertheless, there is a remarkable influence of $\Delta G_{\mathrm{et}}{ }^{\circ}$ on the radical yield observed, such that $\Phi_{\mathrm{r}}$ increases when $\Delta G_{\mathrm{et}}{ }^{\circ}$ becomes more negative. Moreover, when $\ln \Phi_{\mathrm{r}}$ vs. $\Delta G_{\mathrm{et}}{ }^{\circ}$ is plotted (Figure 4) the linear correlation

$$
\ln \Phi_{\mathrm{r}}=b+m \Delta G_{\mathrm{et}}{ }^{\circ}
$$

with $m=-3.2 \mathrm{eV}^{-1}$ and $b=-5.7$, is obtained comprising both amines and methoxy compounds.

Quenching with High Donor Concentrations. Whereas with low quencher concentrations $\left(<5 \times 10^{-2} \mathrm{M}\right)$ the radical yield observed in a flash experiment is proportional to the percentage of fluorescence quenching, a fact that allows us to extrapolate the radical yield in this concentration range to $100 \%$ singlet quenching (cf. eq 9), this does not hold any longer at high quencher concentrations. Especially in the case of electron donors with low oxidation potential (amines), the radical yield passes through a maximum at about $10^{-1} \mathrm{M}$ donor concentration and shows a drastic decrease at higher donor concentrations (cf. Figure 5). Along with this decrease of the radical yield goes a change in the dye absorption spectrum, characterized by a broadening of the long-wavelength absorption with increasing donor concentration. This observation indicates that there is a ground-state interaction between dye and electron donor at high donor concentrations, which may be assumed to be due to electron donor-acceptor 


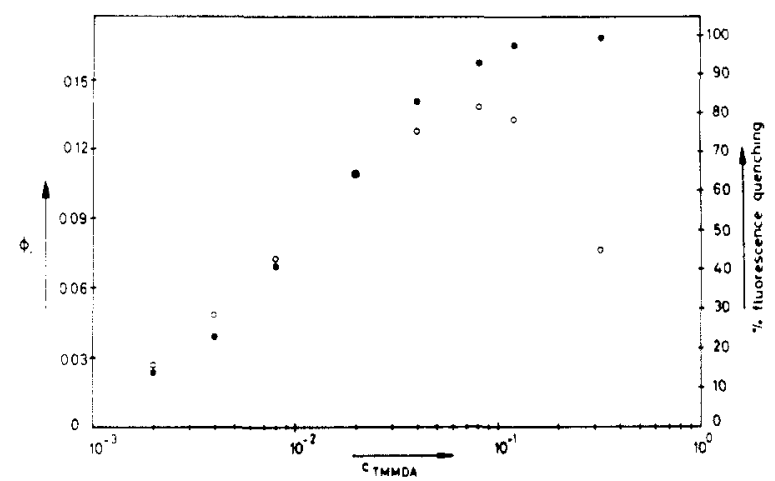

Figure 5. Observed radical yleld $\Phi_{\mathrm{r},[\mathrm{D}]}(\mathrm{O})$ and percentage of fluorescence quenching (O) as a function of donor concentration (donor TMMDA). The relative scaling of the vertical axes was chosen so as to obtain coincident points for radical yield and fluorescence quenching at donor concentration $2 \times 10^{-2} \mathrm{M}$, where $\Phi_{r}$ was determined experimentally according to eq 12 .

(EDA) complex formation. Obviously the radical yield when these EDA complexes are excited is lower than when excited dye singlets are dynamically quenched by donor molecules.

\section{Discussion}

Our discussion of the $\Delta G_{\mathrm{et}}{ }^{\circ}$ dependence of the freeradical yield will be based on the general reaction scheme (Scheme I) described in the Introduction. As was pointed out there, the radical yield should be critically dependent on the nature of the primary electron-transfer product, [3], and the peculiar properties of the processes [3] $\rightarrow$ [4] and $[3] \rightarrow[0]$, which represent the formation of free radicals and deactivation to a lower electronic state of the acceptor, respectively. If the relaxed charge-transfer state is energetically above the locally excited dye triplet (this is the case for donors 19-24) a charge-transfer induced intersystem crossing to this state may occur (cf. Figure 3).

Such processes have indeed been observed for some oxonine/electron donor systems. ${ }^{9}$ For the systems investigated in the present work, however, this mechanism may be neglected. Nanosecond laser-flash experiments ${ }^{26}$ have shown that with donors of the type applied in this work the yield of induced triplet formation is typically $10^{-3}$, which is clearly less than the lowest radical yield observed in the present investigation. Even if all triplets were converted to radicals in a subsequent electron-transfer reaction with the donor, this mechanism would contribute negligibly to the radical yield. Therefore, in the following, we shall have to identify stage [0] in Scheme I with the singlet ground state of the donor-acceptor pair.

We shall now critically examine the nature of electron. transfer product [3] in the light of the linear free-enthalpy relationship for $\ln \Phi_{\mathrm{r}}$ described above.

The Radical Pair Mechanism. In accordance with the original work of Rehm and Weller ${ }^{3}$ the primary electrontransfer product [3] in Scheme I is considerd to be a relaxed, solvent-shared radical pair with the possibility of dissociating to the free, statistically distributed radicals [4] or of recombining to the singlet ground state [0] or to the initial excited encounter pair [2]. The mechanism is represented in Scheme II with symbols appropriate to the present systems, the index $\mathrm{s}$ indicating equilibrium solvation of the respective species.

Since we determined the radical yield $\Phi_{\mathrm{r}}$ in a way as to refer to complete singlet quenching and immediately after

(25) M. Born, Z. Physik, 1, 45 (1920)

(26) E. Vogelmann, unpublished results.
Scheme II

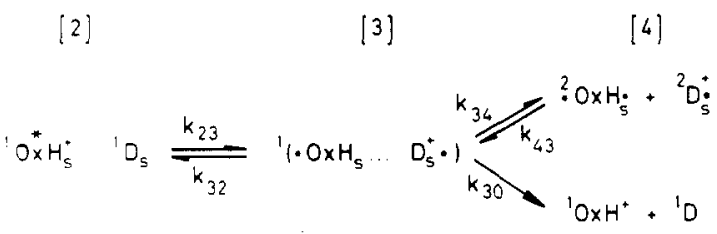

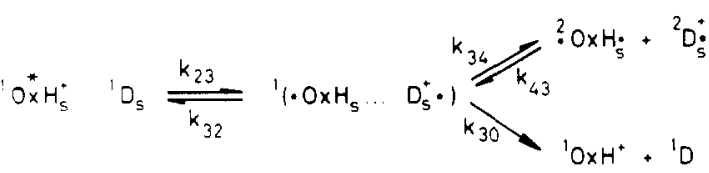

[0]

Scheme III

$$
\begin{aligned}
& \text { 13] [3] }
\end{aligned}
$$

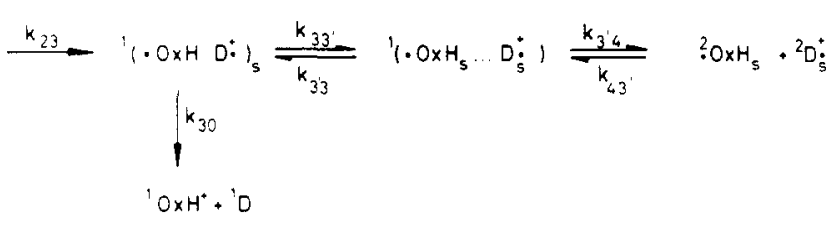

$$
\begin{aligned}
& \text { [0] }
\end{aligned}
$$

the excitation flash when homogeneous recombination of free radicals $(4 \rightarrow 3)$ is negligible, $\Phi_{\mathrm{r}}$ depends only on the rate constants $k_{34}$ and $k_{30}$ as given by eq 1 .

If species [3] is in fact a solvent-shared radical pair, the formation of free, homogeneously distributed radicals [4] is not hindered by Coulomb attraction nor by a specific electronic interaction as would be typical for an exciplex. Thus one should expect $k_{34}$ to be diffusion controlled and independent of $\Delta G_{\mathrm{et}}{ }^{\circ}$ (equivalent to $\Delta G_{23}{ }^{\circ}$ in the case of Scheme II).

The reaction $3 \rightarrow 0$ represents a spin-allowed electrontransfer reaction with $\Delta G_{30}{ }^{\circ}$ given by

$$
\Delta G_{30}{ }^{\circ}=-E_{00}\left({ }^{1}{ }^{\circ} \mathrm{OxH}^{+}\right)-\Delta G_{\text {et }}{ }^{\circ}
$$

Therefore $k_{30}$ can be expected to be $\Delta G_{\text {et }}{ }^{\circ}$ dependent. From Table I and eq 17 it follows that $\Delta G_{30}{ }^{\circ}$ ranges from -1.95 to $-0.52 \mathrm{eV}$, i.e., it is negative for all systems investigated. The influence of $\Delta G_{30}{ }^{\circ}$ and hence of $\Delta G_{\mathrm{et}}{ }^{\circ}$ on $k_{30}$ may be estimated by two different approaches: (a) If we look upon the reaction $3 \rightarrow 0$ as an electron-transfer reaction, we may apply a heuristic relation between $k_{30}$ and $\Delta G_{30}{ }^{\circ}$ established by Rehm and Weller, ${ }^{3}$ according to which $k_{30}$ should become $\Delta G_{30}{ }^{\circ}$ dependent only if $\Delta G_{30}^{\circ}$ $\gtrsim-0.4 \mathrm{eV}$. This is outside the $\Delta G_{30}{ }^{\circ}$ range covered by our systems, so we should have $k_{30}$ almost constant. This conclusion is, however, at variance with our results, which demand that either $k_{34}$ or $k_{30}$ or both be $\Delta G_{\text {et }}{ }^{\circ}$ dependent. (b) Since the reaction $3 \rightarrow 0$ is strongly exothermic, it can be treated by the methods used in the theory of radiationless processes leading to the well-known result of the "energy gap law", ${ }^{8}$ which would require that $k_{30}$ should increase as $\Delta G_{30}^{\circ}$ becomes less negative (equivalent to a decreasing energy gap). From this argument we expect that $k_{30}$ should increase when $\Delta G_{\text {et }}{ }^{\circ}$ becomes more negative, leading to a decrease of the radical yield $\Phi_{r}$, which again is at variance with our observations. Hence a pure radical-pair mechanism as defined by Scheme II must be excluded for the present systems.

The Exciplex Mechanism. A number of effects observed in the electron-transfer quenching of the triplets of thionine $^{27}$ and thiopyronine, ${ }^{6}$ dyes similar to oxonine, strongly suggest that in these cases triplet exciplexes are formed as the primary quenching products in the weakly exothermic region of the electron transfer. From the analysis of a recently discovered novel type of magnetic field effect ${ }^{28}$

(27) (a) U. Steiner, G. Winter, and H. E. A. Kramer, J. Phys. Chem., 81, 1104 (1977). (b) U. Steiner and G. Winter, Chem. Phys. Lett., 55, 364 (1978). 
it is obtained that such triplet exciplexes have a dissociation lifetime of about $1 \mathrm{~ns}$, probably due to resonance interaction bonding. One might suspect that in the present case of oxonine singlet quenching, too, an exciplex intermediate is involved. Thus we may rewrite Scheme I again (Scheme III) where now an exciplex $\left({ }^{1}\left(.0 x \mathrm{H} \mathrm{D}^{+}\right)_{s}\right)$ takes the place of intermediate 3 . In this case formation of free radicals requires dissociation of the singlet exciplex, which we assume here to be a solvated and relaxed species, yielding a geminate, solvent-shared radical pair [ $\left.3^{\prime}\right]$, the final dissociation of which is assumed to be a very efficient process so that in such a scheme the radical yield is mainly determined by the competition of exciplex dissociation $\left(k_{33}\right)$ and its deactivation to the ground state $\left(k_{30}\right)$, and we may write

$$
\Phi_{\mathrm{r}} \propto \frac{k_{33^{\prime}}}{k_{30}+k_{33^{\prime}}}
$$

For the triplet exciplexes between thionine or thiopyronine with halogen anilines ${ }^{6,27}$ it has been observed that with increasing nuclear charge of the halogen substituent, i.e., with increasing spin-orbit coupling, the yield of radicals is markedly reduced due to an enhancement of the process corresponding to $3 \rightarrow 0$ in Scheme I, which in the triplet case represents an intersystem crossing process. Singlet exciplexes with oxonine might be regarded in a sense as a limiting case of such triplet exciplexes with very strong spin-orbit coupling, so that extrapolating the iodine heavy-atom effect on the radical yield in the triplet exciplexes by one order of magnitude in spin-orbit coupling might give an order of magnitude estimate for the expected radical yield of a corresponding singlet exciplex. A radical yield of about $1 \%$ would result in the weakly exothermic region if the dissociation rate constant were the same as for triplet exciplexes. As already mentioned above, if no Coulomb attraction is present, the stability of an exciplex of the type involved in Scheme II with respect to dissociation into radicals is probably determined by a resonance interaction between the locally excited state and the pure charge-transfer state. If the latter is below the locally excited state its energy is lowered by this interaction by an amount of $U_{\text {stab }}$ given by eq $19^{29}$ where $\beta$ is a resonance

$$
U_{\text {stab }}=\left(\Delta E / 4+\beta^{2}\right)^{1 / 2}-\Delta E / 2
$$

integral and $\Delta E$ is the energy difference between the locally excited state and the pure charge-transfer state. It is obvious that $U_{\text {stab }}$ represents a $\Delta G_{\text {et }}{ }^{\circ}$-dependent contribution to the activation energy of exciplex dissociation. Since $U_{\text {stab }}$ decreases when the energy of the pure charge-transfer state is lowered, eq 19 seems to provide an explanation of the $\Delta G_{\mathrm{et}}{ }^{\circ}$ dependence of $\Phi_{\mathrm{r}}$.

Such an argument has been forwarded by Tamura et al. ${ }^{30}$ to explain the $\Delta G_{e t}{ }^{\circ}$ dependence of the radical yield in electron-transfer quenching of methylene blue triplet by ferrocenes. In the following we shall critically examine the implication of this mechanism on our present results.

In our opinion the energy difference $\Delta E$ must not be directly equalized to the free-enthalpy change $\Delta G_{\mathrm{et}}{ }^{\circ}$ for reasons that are illustrated in Figure 6 . In calculating the resonance interaction between two electronic states, one has to take care that in both states the nuclear coordinates are identical. This is of special importance with respect

(28) U. Steiner, Chem. Phys. Lett., 74, 108 (1980). U. Steiner, Ber. Bunsenges. Phys. Chem., 85, 228 (1981).

(29) A. Weller in "The Exciplex", M. Gordon and W. R. Ware, Eds., Academic Press, New York, 1975, p 24.

(30) S. Tamura, K. Kokuchi, H. Kokubun, and A. Weller, Z. Phys. Chem. (Wiesbaden), 121, 165 (1980).

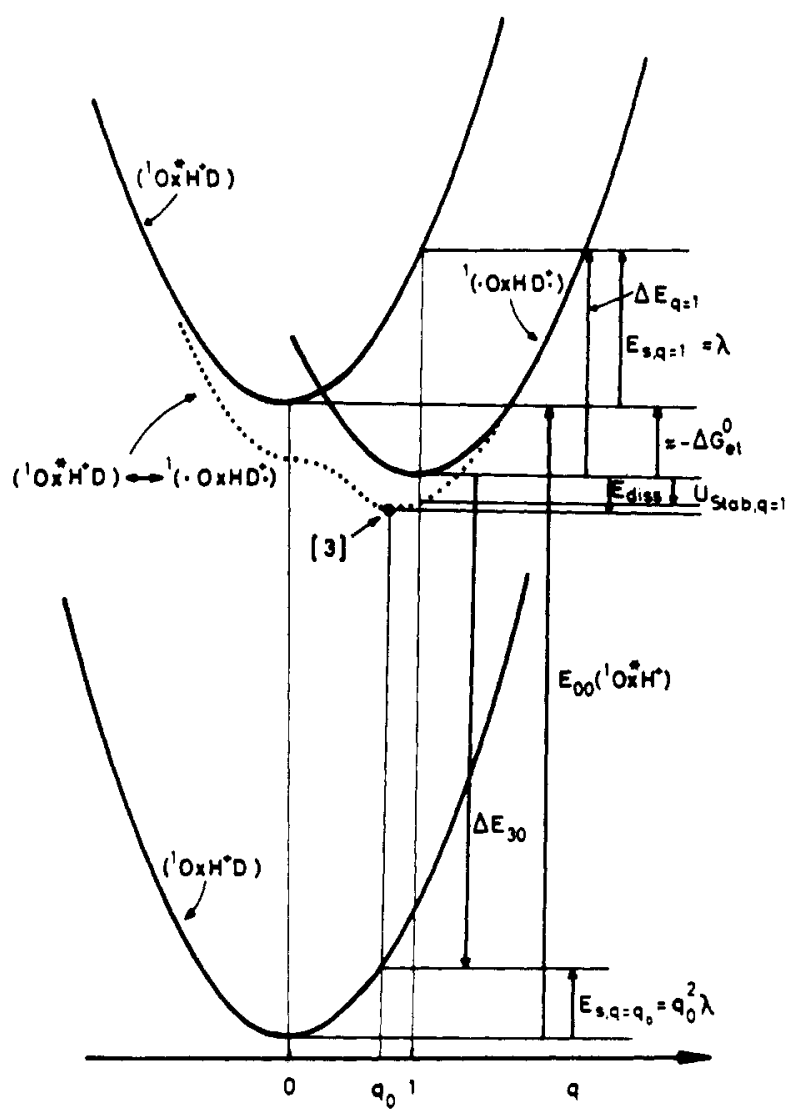

Flgure 6. Potential energy of various states as a function of solvent orientation parameter $q$, defined to correspond to a solvent orlentation in equilibrium with the hypothetical charge distribution $\left(0 \times H^{(1-q)} D^{9}\right)$. Potential energy curves are glven for different charge distrlbutions and states as indicated in the figure. The dotted curve refers to the exciplex that is stabilized with respect to the lower of the localized charge configurations ( $\left.{ }^{1 *} \mathrm{OxH}^{+} \mathrm{D}\right)$ and ${ }^{1}\left(\cdot \mathrm{O} \times \mathrm{HD}^{+}\right)$by the resonance interaction energy calculated according to eq 19 . The point marked by [3] corresponds to an exclplex with equillbrium solvent orientation (cf. Scheme II). The various arrows in the dlagram illustrate the meaning of the energetic quantities used in eq 19-22.

to the solvent nuclei. Since the charge distribution of the reaction complex is different in the locally excited $\left({ }^{1 *} \mathrm{OxH}^{+} \mathrm{D}\right)$ and charge-transfer ${ }^{1}\left(\cdot \mathrm{OxH} \mathrm{D}^{+}\right)$electronic configuration, the solvent equilibrium orientation will also be different in these configurations.

Therefore the energy gap between the two states entering into eq 19 must not be identified with the equilibrium energy difference-which would be fairly well represented by $\Delta G_{\mathrm{et}}{ }^{\circ}$--but is composed of $\Delta G_{\mathrm{et}}{ }^{\circ}$ and a resolvation energy contribution $E_{8}$. In order to forward quantitative arguments, we introduce a solvent orientation parameter $q$, describing the equilibrium solvent orientation for a fixed charge distribution $\mathrm{OxH}^{1-q} D^{q}$. We assume a quadratic dependence of the resolvation energy on $q$

$$
E_{\mathrm{s}}=\lambda q^{2}
$$

where $\lambda$ is a parameter depending on the solvent polarity and the radii and distance of the interacting molecules $\mathrm{OxH}^{+}$and $\mathrm{D} .{ }^{31}$ With these definitions the energy gap $\Delta E$ to be used in eq 19 becomes a function of $q$, given by

$$
\Delta E(q)=-\Delta G_{\mathrm{et}}{ }^{\circ}+(2 q-1) \lambda
$$

From a calculation of the stabilization energy $U_{\text {stab }}$ as a function of $q$, the solvation energy curve of the exciplex is obtained (cf. Figure 6). The minimum $q_{0}$ of this curve may be easily determined by using an iterative procedure

(31) R. A. Marcus, J. Chem. Phys., 24, 966 (1956). 

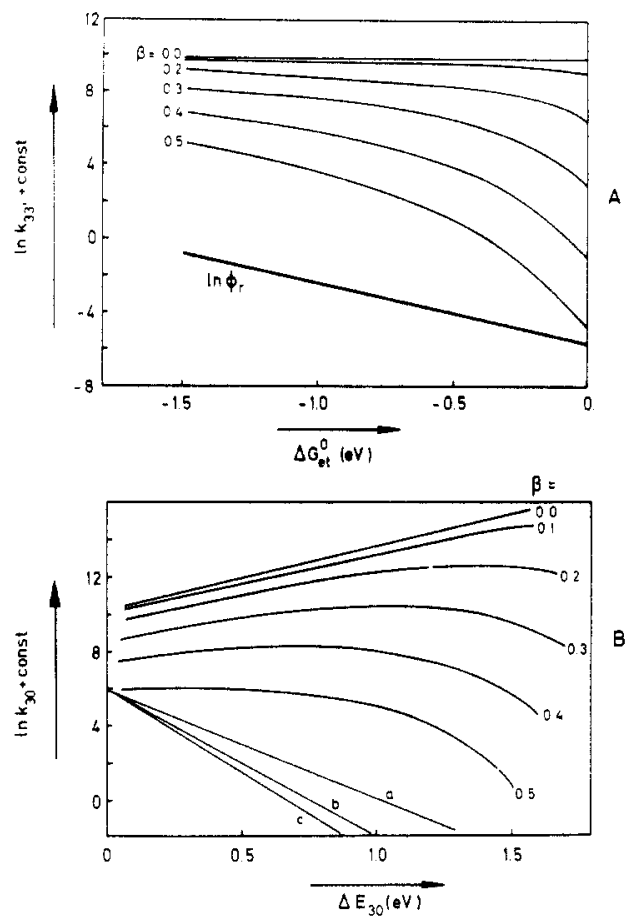

Flgure 7. (A) Dependence of In $k_{33^{\prime}}$ on $\Delta G_{e t}{ }^{\circ}$ calculated by using eq $19-21$ with various values $(\theta \mathrm{V})$ of the resonance integral $\beta$. Also shown is the dependence of $\ln \Phi_{r}$ on $\Delta G_{\text {ot }}{ }^{\circ}$ as found in the experiments. (B) In $k_{30}$ obtained from eq 26 for the same $\beta$ values as in $A$ as a function of $\Delta E_{30}$, the electronic energy gap for radiationiess deactivatoin of the exciplex. The straight lines $a, b$, and $c$ reter to previous observations of the energy gap law, described in ref 34.

based on the condition that the effective charge of $D$ in the exciplex (corresponding to the square of the coefficient of the charge-transfer state in the exciplex wave function) be equal to $q$. From Figure 6 it follows that the dissociation energy $E_{\text {diss }}$ of the exciplex into free radicals is given by

$$
E_{\text {diss }}=U_{\text {stab }}\left(q_{0}\right)-\left(1-q_{0}\right)^{2} \lambda
$$

Following Tamura et al. ${ }^{30}$ we may set

$$
k_{33^{\prime}}=A_{33^{\prime}} \exp \left(-E_{\text {diss }} / R T\right)
$$

or

$$
\ln \left(k_{33^{\prime}}\right)=a-E_{\text {diss }} / R T
$$

where $A_{33^{\prime}}$ is a factor independent of $\Delta G_{\mathrm{et}}{ }^{\circ}$ and $a=\ln \mathrm{A}_{33^{\prime}}$. We have calculated $\ln \left(k_{33^{\prime}}\right)$ as a function of $\Delta G_{\text {et }}{ }^{\circ}$ using eq 19 and 21 with $\lambda=0.435 \mathrm{eV}(\cong 10 \mathrm{kcal})^{32}$ and a series of values for $\beta$, covering a reasonable range from 0.1 to 0.5 $\mathrm{eV}^{33}$ The resulting curves are plotted in Figure 7A.

From eq 18 we obtain

$$
\ln \left(1 / \phi_{\mathrm{r}}-1\right)=\mathrm{const}-\ln \left(k_{33^{\prime}} / k_{30}\right)
$$

For values $\Phi_{\mathrm{r}} \ll 1$, valid for almost all of our systems, eq 25 is approximated by

$$
\ln \Phi_{\mathrm{r}} \approx \text { const }+\ln \left(k_{33^{\prime}} / k_{30}\right)
$$

Thus the interpretation of the diagram in Figure 4 would

(32) U. Steiner, "Schweratome als molekulare Sonden zum Nachweis und Studium des Verhaltens von Triplettexciplexen", Hochschulverlag, Freiburg, 1979.

(33) This range has been reasonably assumed by S. Iwata, I. Tanaka and $\mathbf{S}$. Nagakura in "The Triplet State". Proceedings of the International Symposium, Beirut, Lebanon, 1967, A. B. Zahlan, Ed., p 433, Cambridge University Press, 1967. A value in the order of $0.15 \mathrm{eV}$ has been calculated theoretically for the anthracene diethylaniline exciplex by $\mathrm{H}$. Beens and A. Weller, "Organic Molecular Photophysics", Vol. 2, J. B. Birks, Ed., Wiley, London, 1975, p 159. be that it is the quantity $\ln \left(k_{30} / k_{33^{3}}\right)$ that correlates linearly with $\Delta G_{e t}{ }^{\circ}$, and we may use this correlation (eq 16) together with eq 24 and 26 to calculate the variation of in $\left(k_{30}\right)$ as a function of $\Delta G_{\text {et }}{ }^{\circ}$ or, what is more interesting, as a function of $\Delta E_{30}$ related to $\Delta G_{\mathrm{et}}{ }^{\circ}$ (cf. Figure 6) by

$$
\Delta E_{30}=E_{00}\left({ }^{1} * \mathrm{OxH}^{+}\right)+\Delta G_{\mathrm{et}}{ }^{\circ}-E_{\mathrm{diss}}-q_{0}{ }^{2} \lambda
$$

The values of $\ln k_{30}$ obtained in this way are plotted vs. $\Delta E_{30}$ in Figure 7B for the different $\beta$ values used in Figure 7A. From these curves we may decide now whether the exciplex mechanism is physically sound, since they should reflect the energy gap law of radiationless transitions. In fact none of the curves shown in Figure 7B corresponds to the typical energy-gap-law behavior that is normally represented by an almost straight line in a $\ln \left(k_{n r}\right) / \Delta E$ diagram with a slope of about $6-9 \mathrm{eV}^{-1} \cdot{ }^{34}$ The curves obtained are far from linear and in large regions even show anti-energy-gap-law behavior.

Thus, whereas the exciplex mechanism may account for the order of magnitude of the radical yield in the weakly exothermic free-enthalpy region, it fails to explain the $\Phi_{\mathrm{r}}$ dependence on $\Delta G_{\mathrm{et}}{ }^{\circ}$ over the wide range of $\Delta G_{\mathrm{et}}{ }^{\circ}$ investigated.

In a discussion of the possible role of exciplexes in the radical formation mechanism it is of interest to consider the consequences of the results presented in Figure 5. These findings indicate that in case of the amine donors the radical yield is much lower from the excited EDA complex that they are when dynamically quenching the excited singlet oxonine.

Mataga et al.,12 when investigating radical formation from excited EDA complexes, found a decrease of the radical yield when lowering the energy of the chargetransfer state in qualitative accord with the energy gap law. The low radical yields from the excited EDA complexes in our systems are in line with these observations since in our case the energy gap is smaller than in the lower limiting system investigated by Mataga.

Gaweda and Prochorow ${ }^{35}$ found examples of systems where the typical exciplex fluorescence in liquid solution can be produced either by exciting the ground-state EDA complex or by dymamically quenching a locally excited singlet state. In fact it is easy to imagine that the specific geometric requirement of exciplex formation is similar to the configuration preferred by the ground-state EDA complex since bonding of both depends on optimum electronic overlap. Thus if we assume that electronic relaxation of the excited EDA complex in our systems passes through the exciplex configuration (intermediate 3 in Scheme II), we have to conclude from Figure 5 that the radical yield $\Phi_{r}$ is less from exciplex dissociation (Figure $5, c>10^{-1} \mathrm{M}$ ) than from dynamic singlet quenching (extrapolated from $c \leq 5 \times 10^{-2} \mathrm{M}$ to complete quenching). Therefore dynamic singlet quenching must be due mainly to a mechanism excluding an exciplex of the properties attributed to intermediate 3 in Scheme II.

A Switchover Mechanism. In the preceding discussion we have seen that neither the pure radical pair mechanism nor the exciplex mechanism could provide a satisfactory explanation of the observed free-enthalpy relation for $\ln$ $\Phi_{r}$. Whereas a solvent-shared radical pair without Coulomb attraction can be expected to give high radical yields that are quite insensitive to $\Delta G_{\text {et }}{ }^{\circ}$, the radical yield for

(34) (a) W. Siebrand, J. Chem. Phys., 44, 4055 (1966). (b) H. Dreeskamp, E. Koch, and M. Zander, Ber. Bunsenges. Phys. Chem., 78, 1328 (1974). (c) S. Murata, C. Iwanaga, T. Toda, and H. Kokubun, Chem. Phys. Lett., 15, 152 (1972).

(35) E. Gaweda and J. Prochorow, Chem. Phys. Lett., 30, 155 (1975). 
Scheme IV

[3]

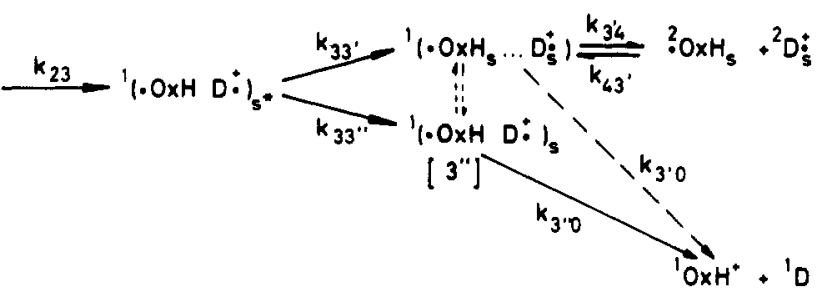

[0]

singlet exciplexes in the present systems should not exceed a value of about $1 \%$ for weakly negative $\Delta G_{\text {et }}{ }^{\circ}$ and become even smaller in the more negative $\Delta G_{\text {et }}{ }^{\circ}$ region. Thus, while none of these mechanisms is sufficient on its own, it is tempting to assume that both intermediates may be formed competitively with the branching ratio between them being $\Delta G_{\text {et }}{ }^{\circ}$ dependent.

The kinetic representation of such a third specialization of the general Scheme I is given in Scheme IV. Here the primary electron-transfer product [3] can be described as a loose charge-transfer complex with incomplete solvent relaxation, expressed by the index $\mathrm{s}^{*}$, where part of the free electron-transfer enthalpy $\Delta G_{\mathrm{et}}{ }^{\circ}$ can be thought of as being present in the solvent "strain". In the course of the solvent relaxation, species 3 may transform either to a solvent-shared radical pair [3'] or to an exciplex with equilibrium solvation [ $\left.3^{\prime \prime}\right]$, the behavior of which shall be assumed as described in the last section. The assumption that a charge-transfer state, unrelaxed with respect to solvation, is the precursor of the solvent-shared radical pair and the equilibrated exciplex has been suggested by Mataga et $a .^{36}$ in order to explain differences in the time dependence of photoinduced current and exciplex fluorescence in the quenching of the excited pyrene singlet by $\mathrm{N}, \mathrm{N}$-dimethylaniline.

A direct competition between formation of a solventshared radical ion pair and a (fluorescing) exciplex has been also proposed by Weller et al. ${ }^{37}$ to explain the different solvent dependence of exciplex fluorescence quantum yield and lifetime.

Let us now consider in detail how the mechanism represented in Scheme IV can explain the observed linear free-enthalpy relation. In this case the radical yield under conditions of complete dynamic singlet quenching is given by eq 28 , where $\Phi_{3^{\prime} 4}$ denotes the dissociation yield of the

$$
\Phi_{\mathrm{r}}=\frac{k_{33^{\prime}}}{k_{33^{\prime}}+k_{33^{\prime \prime}}} \Phi_{3^{\prime} 4}
$$

geminate solvent-shared radical pair $\left[3^{\prime}\right]$ into free radicals. It is reasonable to assume that for such a species the dissociation yield into free radicals is $\gtrsim 0.5$ since reencounters of the pair are not favored by Coulomb attraction. Furthermore, since $k_{3^{\prime} 0}$ represents the rate constant of an exothermic electron-transfer reaction it should be weakly dependent on $\Delta G_{3^{\prime} 0}$. Thus we may assume that $\Phi_{3^{\prime} 4}$ is fairly

(36) Y. Taniguchi, Y. Nishina, and N. Mataga, Bull. Chem. Soc. Jpn. 45, 764 (1972). H. Masuhara, T. Hino, and N. Mataga, J. Phys. Chem. 79,994 (1975).

(37) H. Knibbe, K. Röllig, F. P. Schäfer, and A. Weller, J. Chem. Phys., 47, 1184 (1967). constant in the series of donors investigated. Equation 28 may be transformed to

$$
\ln \left(\Phi_{3^{\prime} 4} / \Phi_{\mathrm{r}}-1\right)=\ln k_{33^{\prime \prime}} / k_{33^{\prime}}
$$

which under the assumption that $0.5 \approx \Phi_{3^{\prime} 4} \gg \Phi_{\mathrm{r}}$, valid for the great majority of systems in Table I, is approximately equal to

$$
\ln \Phi_{\mathrm{r}} \approx \ln k_{33^{\prime}} / k_{33^{\prime \prime}}+\text { const }
$$

so that, in terms of the present mechanism, the observed linear free-enthalpy relation has to be interpreted by the combination of eq 16 and 30 , yielding

$$
\ln k_{33^{\prime}} / k_{33^{\prime \prime}}=\text { const }+m \Delta G_{\mathrm{et}}{ }^{\circ}
$$

i.e., the logarithm of the branching ratio between radical pair formation and relaxed exciplex formation should be linearly dependent on $\Delta G_{\text {et }}{ }^{\circ}$. If we assume that $k_{33^{\prime \prime}}$, the rate constant of relaxation to the equilibrated exciplex, is independent of the free enthalpy, which is stored in the solvent strain of the state ${ }^{1}\left(\cdot \mathrm{OxH} \mathrm{D}{ }^{+}\right)_{\mathrm{B}^{*}}$, it would be the rate constant $k_{33^{\prime}}$ of the charge-transfer-complex separation by solvent molecules alone, reflecting the $\Delta G_{\mathrm{et}}{ }^{\circ}$ dependence of the branching ratio. In $\left(k_{33^{\prime}}\right)$ is determined by an activation energy $E_{33^{*}}{ }^{*}$ according to

$$
\ln k_{33^{\prime}}=\text { const }-E_{33^{\prime}}{ }^{*} / \mathrm{RT}
$$

Assuming $k_{33^{\prime \prime}}$ to be independent of $\Delta G_{\mathrm{et}}{ }^{\circ}$ would in fact mean that $E_{33^{\prime}}$ depends linearly on $\Delta G_{\mathrm{et}}{ }^{\circ}$ according to

$$
E_{33^{\prime}}{ }^{*} / R T=\text { const }-m \Delta G_{\mathrm{et}}{ }^{\circ}=\text { const }+\alpha \Delta G_{\mathrm{et}}{ }^{\circ} / R T
$$

where we introduce a factor $\alpha=-m R T$, denoting the fraction of $\Delta G_{\mathrm{et}}{ }^{\circ}$ that is used to lower the activation energy $E_{33^{\prime}}{ }^{*}$. Using the numerical result for $m\left(-3.2 \mathrm{eV}^{-1}\right)$, we obtain for $\alpha$ a value of 0.09 , i.e., about $10 \%$ of $\Delta G_{\mathrm{et}}{ }^{\circ}$ would be used by the solvent shell to lower the activation energy $E_{33^{\prime}}{ }^{*}$ for separating the reactants of the electron-transfer reaction by solvent molecules, a value that does not seem unreasonable.

\section{Conclusion}

It is common to both the "radical pair" and the "exciplex" mechanism that the radical yield is determined by the competition of a dissociation reaction and a strongly exothermic electron retransfer or radiationless transition with charge-transfer character. As far as our present understanding of these processes and their $\Delta G_{\mathrm{et}}{ }^{\circ}$ dependence goes, they cannot explain the $\Delta G_{e t}{ }^{\circ}$ dependence of the radical yield observed in this work. The "switchover" mechanism introduces the solvent-shared radical pair and the relaxed exciplex as intermediates formed competitively (not consecutively as in the exciplex mechanism) from a nonrelaxed primary charge-transfer intermediate. In the "switchover" mechanism both competing reactions $\left(k_{33^{\prime}}\right.$ and $k_{33^{\prime \prime}}$ in Scheme IV) determining the radical yield represent conformational (solvent and exciplex) relaxation processes, whereas in the pure "radical pair" and the "exciplex" mechanism one of the relevant processes corresponds to electronic relaxation to the ground state.

Acknowledgment. Financial support by the Deutsche Forschungsgemeinschaft and the Fonds der Chemischen Industrie is gratefully acknowledged. 